\title{
Energy-aware Restricted Access Window Control with Retransmission Scheme for IEEE 802.11ah (Wi-Fi HaLow) based Networks
}

\author{
Yanru Wang*, Kok Keong Chai*, Yue Chen*, John Schormans* and Jonathan Loo ${ }^{\dagger}$ \\ ${ }^{*}$ School of Electronic Engineering and Computer Science, Queen Mary University of London, London, UK \\ Email: \{yanru.wang, michael.chai, yue.chen, j.schormans\}@qmul.ac.uk \\ ${ }^{\dagger}$ Department of Computer Communications, School of Sciences and Technology, Middlesex University, London, UK \\ Email: J.Loo@mdx.ac.uk
}

\begin{abstract}
Restricted Access Window (RAW) has been introduced to IEEE 802.11ah MAC layer to decrease collision probability. However, the inappropriate application of RAW for different groups of devices would increase uplink energy consumption and decrease data rate. In this paper, we study an energy efficient RAW optimization problem for IEEE 802.11ah based uplink communications. We first present a novel retransmission scheme that utilizes the nextt empty slot to retransmit for collided devices, and formulate the problem based on overall energy consumption and the data rate of each RAW by applying probability theory and Markov Chain. Then, we derive the energy efficiency of the uplink transmission. Last but not the least, an energy-aware window control algorithm to adapt the RAW size is proposed to optimize the energy efficiency by identifying the number of slots in each RAW for different group scales. Simulation results show that our proposed algorithm outperforms existing RAW on uplink energy efficiency and delivery ratio.
\end{abstract}

Index Terms-IEEE 802.11ah, MAC, RAW, uplink energy efficiency, retransmission

\section{INTRODUCTION}

The emerging concept of Internet-of-Things (IoT) has brought a new way to communicate and a major influence on human life style. It indicates there will be a large number of sensors and devices in such networks [1] [2] [3]. However, the existing wireless technologies such as RFID, ZigBee, Bluetooth etc. can not accommodate this amount of devices with high throughput over a large transmission range [4].

One of the latest wireless communication technologies that been proposed for IoT applications is the Low Power WiFi [5]. IEEE 802.11ah Wireless LAN standard group has put forward IEEE 802.11ah to support Low Power Wi-Fi, called "Wi-Fi HaLow" [6] [7]. IEEE 802.11ah operates at sub-1 GHz and it can support up to 6000 devices within a network with transmission range up to $1 \mathrm{~km}$ at the rate of more than 100 kbps [8].

To deal with high contention of high density of devices, Restricted Access Window (RAW) is a new MAC layer feature that used in Low Power Wi-Fi to reduce collision probability. It limits a set of devices that can access the channel at a time and spreads their attempts over a long period of time to enable the efficient network communication of a high number of devices
[6] [9]. RAW consists of multiple equal time slots, where each slot is selected by devices or assigned to a group of devices for transmission [4] [10]. Only a subset of devices are allowed to contend at the same time [15] [20] [21]. So all the devices are in active mode only when turning to their related RAW, otherwise would be in doze mode to conserve power.

The existing literature to improve energy efficiency has been studied in depth in many research works, mainly focus on improving successful transmission probability and reducing collision probability. In [10], a new medium access control enhancement algorithm was proposed to determine optimal size of RAW. It used Maximum Likelihood (ML) estimation method to estimate the number devices for uplink access and determine the optimal size of RAW. However, it only involved successful transmission probability without consideration of energy. In [11], the authors introduced Successive Interference Cancellation (SIC) to improve the throughput in a limited time period by sending duplicate packets to different slots to decrase the number of empty slots, but it results in more collisions. In [12] and [13], new algorithms were proposed to save power by estimating target wake-up time for each device to extend doze time. They used probability theory and matrix way, which could monitor different communications scenarios, to build analysis model. However, these algorithms have not embodied the RAW communications mechanism. In [14], low collision probability was achieved through access control to limit the number of devices contending by setting the authentication threshold of the access point (AP), and making a decision by comparing threshold with the numbers that devices randomly generate in the network. It only fitted for authentication stage instead of transmitting and receiving process. In [15], the RAW mechanism is analyzed based on non-cross slot boundary holding scheme. However, it focuses on the holding scheme instead of RAW size and retransmission. In our previous work [16], the optimal uplink energy efficiency is achieve by adaptive RAW duration with aproporite number of devices per group for IEEE 802.11ah based networks. However, it has not considered the retransmission mechanism and there are many empty slots in each RAW. Also it is a static secheme instead of a dynamic RAW for different group sizes. 
The aforementioned literatures laid a solid foundation in improving energy efficiency based on transmission probability and reducing collision for IEEE 802.11ah. Less work has been done to optimize the energy efficiency with joint consideration of RAW size based on clustering size and retransmission control. To address the above joint consideration, we study an optimization problem aiming at maximizing uplink energy efficiency in IEEE 802.11ah based networks. We focus on RAW control with retransmission scheme to alleviate the empty slots waste and increase the amount of data being tranmitted when consuming one Joule energy. The main contribution of this paper are summarized as follows:

1) The novel re-transmission scheme is presented to reuse the empty time slots in one RAW. For random selection in uplink communications, there are some empty slots without devices to choose, while some slots contains more than one device to attempt accessing. Motivated by that, novel retransmission scheme is designed to alleviate idle slots waste, which could decrease the idle listenning comsumption and improve the number of packets transmitted in a certain time period.

2) The accessing process of each time slot in RAW based on novel retransmisson scheme is formulated by probability theory and Markov Chain. As for the novel retransmission scheme, there is dependency between the adjacent slots. The number of devices in a time slot to attempt accessing the channel relies on the state of the previous slot. So probility theory is applied to show the likelihood of different state for one slot and Markov Chain is a good candicate to monitor the dependency.

3) A energy-aware RAW control with retransmission scheme based on group size is proposed to optimize uplink energy efficiency for IoT applications. The scheme is applicable for AP to dynamically estimate the adaptive RAW size for various group size based on the novel retransmission, which is for the collided devices to retranmit its packet efficiently.

4) An optimal RAW duration for IEEE 802.11ah MAC layer is mathematical derived by applying Gradient Descent approach, an efficient optimization tool for the global optimization problem with a large search scale. And the simulation results demonstrate the effect of the RAW control.

The remainder of the paper is organized as follow. Section II describes system model about IEEE 802.11ah MAC uplink communication with RAW mechanism based on novel retransmission scheme. Problem formulation about accessing process of each time slot in RAW based on retransmission is given in Section III. Section IV presents the derivation of optimal solution and energy-aware RAW control algorithm. Section $\mathrm{V}$ provides the simulation results to show the efficacy of algorithm we proposed. Finally, section VI concludes the paper.

\section{SYSTEM MODEL}

We consider a single-hop topology for dense IEEE 802.11ah networks as a single Access Point (AP) surrounded by a high number of devices. In IEEE 802.11ah, RAW groups the devices with consideration of the location, traffic, type, energy saving mode etc., and splits the channel into equal time slots [17]. The AP broadcasts all RAW parameters periodically in the beginning of beacon frame called Target Beacon Transmission Time (TBTT) to inform devices which group they belong to and when the group RAW slot occurs, so all devices in this network keep active and listen in TBTT to obtain scheduling information. Since transmissions outside the RAW slot are not protected from collisions at all, devices would keep in sleep mode to save energy except turning to their RAW to attempt accessing the channel [19]. We assume for a RAW, there are $M$ time slots for $N$ devices limited by lowest and highest Associated Identifier (AID) of devices (a unique ID, through which the AP can indicate its associated devices) [4] [19]. The number of time slots would dynamically change based on the number of devices that need to exchange uplink information in one group. For focusing on MAC layer, we assume that all packets are always transmitted without error due to the channel impairments.

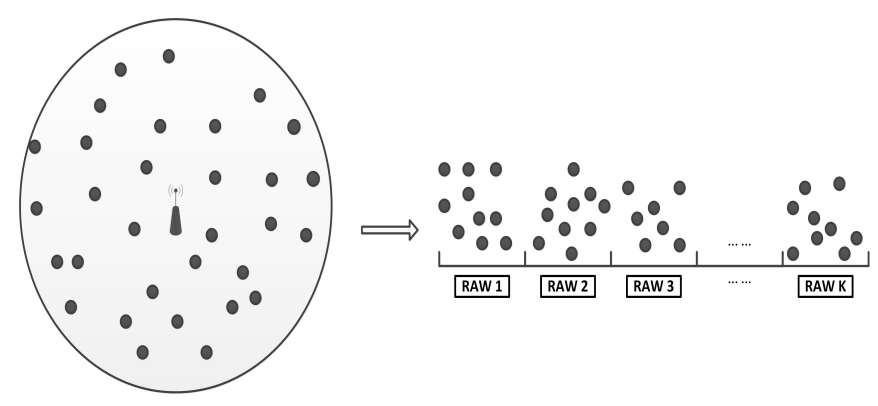

Fig. 1. System model.

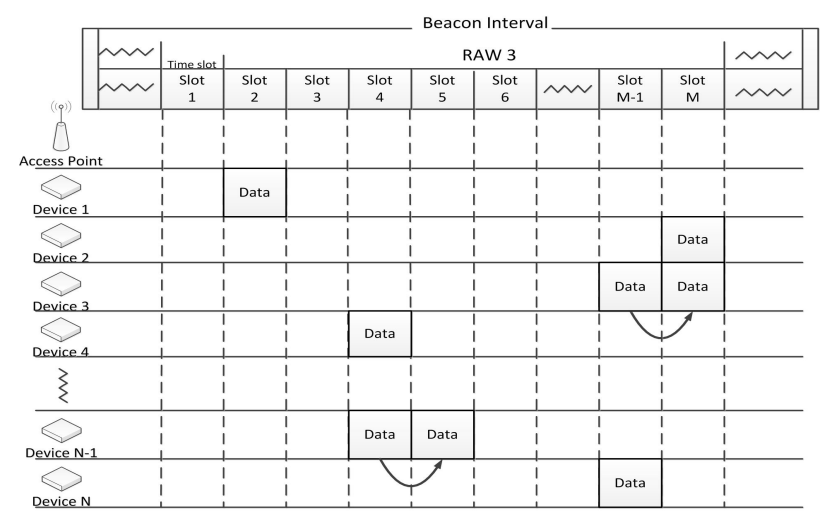

Fig. 2. Operation of RAW.

In IEEE 802.11ah uplink communications, the devices who have buffered data for the AP randomly select a time slot in allocated RAW and attempt to access channel as shown in Fig. 2. If there is only one device in a slot, it could access 
directly as Device 1 transmits packet in Slot 2 directly without contention. When there are more than one device choosing the same time slot, for example, Device 3, Device $\mathrm{N}$ in Slot M-1, and the same for Device 4 and Device N-1 in Slot 4, they would come into the back-off stage to avoid collision by doubling contention window and trying again until reaching the slot boundary. If accessing successfully, device requests uplink communication by sending PowerSave-poll (PS-poll) message to the AP. AP responses with an ACK to confirm connection. After the first handshake, the device transmits buffered data frame and waits for ACK from AP [18]. We assume if not accessing the channel, it would retransmit in the next slot, for example, when Device $\mathrm{N}$ has accessed in Slot M-1, Device 3 retransmits in Slot M, which needs to contend with Device 2, and Device N-1 retransmits in Slot 5 as Device 4 has accessed in Slot 4. But when they can not retransmit successfully in the following slot, they need to retransmit in subsequent allocated RAW. The process is repeated, one RAW by one RAW, until the end of beacon frame.

\section{PROBLEM FORMULATION}

In this section, the process of devices to access channel in IEEE 802.11ah is formulated by probability theory and Markov Chain. Based on that, the energy efficiency is derived.

For uplink communication in MAC layer of IEEE 802.11ah, the number of devices to contend is limited by RAW. Time slots in RAW are selected by devices randomly. Since random selection, the states of each time slot are different, and retransmission makes it depend on the state of previous slot, so Markov Chain is applied to monitor accessing process of each time slot in one RAW.

The way to estimate uplink energy efficiency for one RAW could be divided into four steps:

1) Minitoring the accessing and transmission process in each time slot. And formulating the dependency between adjacent slots.

2) Calculating probability of different states for one device to transmit single packet successfully in one uplink RAW.

3) Estimating the overall energy consumption for one device to attempt accessing the channel for uplink communications and the data rate that could be achieved in one uplink RAW.

4) Formulating energy efficiency with data rate and energy consumption of all uplink devices involved for one RAW.

The process in Markov Chain is represented by three parameters $j, n_{j}, s_{j}$, where $j$ is the index of time slot in one RAW; $n_{j}$ shows there are $n_{j}$ devices to choose the $j t h$ time slot; $s_{j}$ is the number of devices accessing channel successfully, which is illustrated as Fig. 3.

$P_{d}$ is the probability of a certain number of devices to choose this slot, and $P_{s}$ is the probability for accessing sucessfully in this slot.

The transition probability of adjacent states could be denoted as Equation 1, where $P_{i j}$ is the probability to transfer

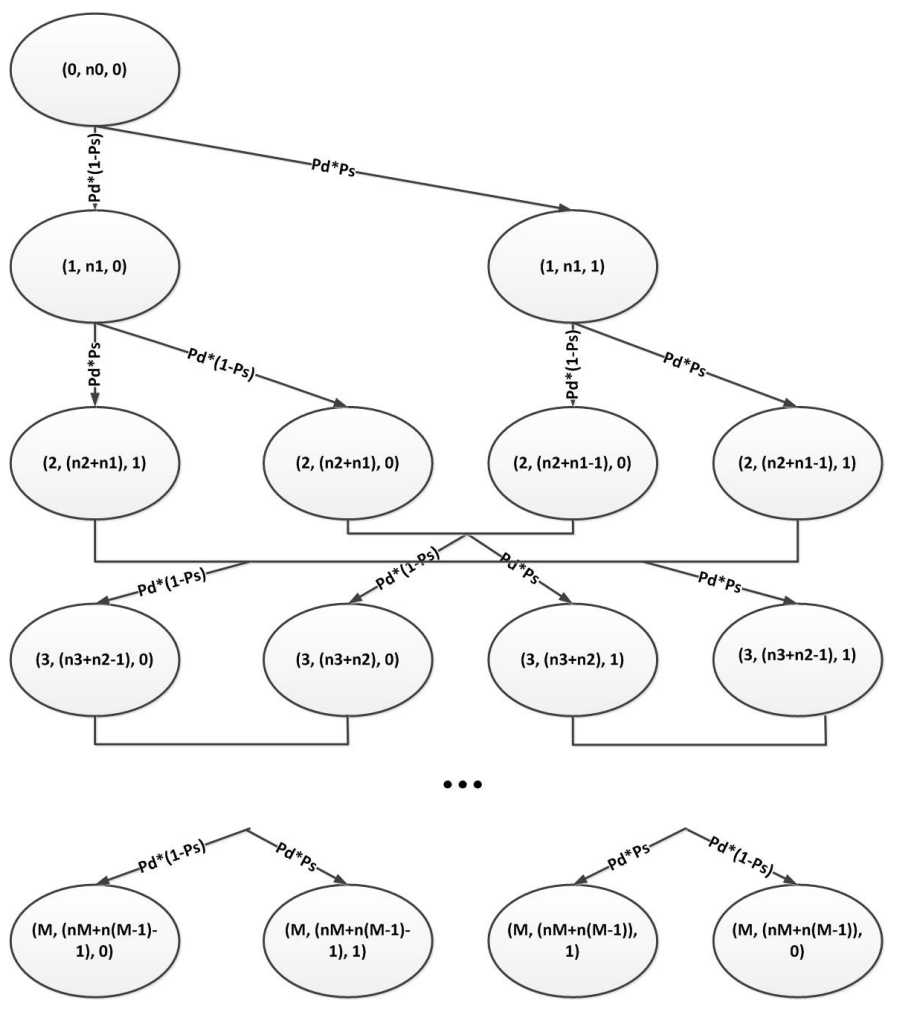

Fig. 3. Markov Chain.

from slot $i$ to slot $j ; \mathrm{P}_{d_{-} n_{j}}$ is the probability of there are $n_{j}$ devices to choose the same time slot; $\mathrm{P}_{s_{-} n_{j}}$ is the probability of successfully accessing in $j t h$ slot for $n_{j}$ devices; $i=0$ is the initial state.

- $\mathrm{P}_{d_{-} n_{j}} \mathrm{P}_{s_{-} n_{j}}$ is the probability of successful transmission in first slot.

- $\mathrm{P}_{d_{-} n_{j}}\left(1-\mathrm{P}_{s_{-} n_{j}}\right)$ is the unsuccessful case in first slot.

- $\mathrm{P}_{d_{-} n_{j}} \mathrm{P}_{s_{-}\left(n_{j}+n_{i}-s_{i}\right)}$ is the probability of transmitting successfully in $j$ th time slot. $\left(n_{i}-s_{i}\right)$ is the number of devices who choose ith slot when selecting randomly, but fail to access the channel and need to attempt to retransmit in $j t h$ slot.

- $\mathrm{P}_{d_{-} n_{j}}\left(1-\mathrm{P}_{s_{-}}\left(n_{j}+n_{i}-s_{i}\right)\right)$ is the probability of unsuccessful transmission in $j$ th time slot.

As for a time slot in RAW, the probability that there are $n_{k}$ devices to choose is

$$
P_{d \_n k}=\left(\begin{array}{c}
N \\
n_{k}
\end{array}\right)\left(\frac{1}{M}\right)^{n_{k}}\left(1-\frac{1}{M}\right)^{N-n_{k}},
$$

where $M$ is the number of time slots contained in one RAW; $N$ is the number of devices that could be involved in one RAW and intend to access the channel; $n_{k}$ is the number of devices to choose the $k$ th time slot.

According to [10], there are two cases for a device to transmit uplink packet successfully in one slot.

- Case 1: a time slot chosen by only one device. This device would transmit a packet successfully without other contending devices. 


$$
P_{i j}= \begin{cases}\mathrm{P}_{d_{-} n_{j}} \mathrm{P}_{s_{-} n_{j}}, & \text { if }\left(i=0,0<n_{j}<N, s_{j}=1\right) \\ \mathrm{P}_{d_{-} n_{j}}\left(1-\mathrm{P}_{s_{-} n_{j}}\right), & \text { if }\left(i=0,0<n_{j}<N, s_{j}=0\right) \\ \mathrm{P}_{d_{-} n_{j}} \mathrm{P}_{s_{-}\left(n_{j}+n_{i}-s_{i}\right)}, & \text { if }\left(i>0,0<n_{j}<\left(N-\sum_{k=0}^{i-1} n_{k}\right), s_{j}=1\right) \\ \mathrm{P}_{d_{-} n_{j}}\left(1-\mathrm{P}_{s_{-}\left(n_{j}+n_{i}-s_{i}\right)}\right), & \text { if }\left(i>0,0<n_{j}<\left(N-\sum_{k=0}^{i-1} n_{k}\right), s_{j}=0\right) \\ 0, & \text { otherwise }\end{cases}
$$

- Case 2: a time slot chosen by multiple devices. The devices will go into back-off stage, and one of them will succeeds in accessing the channel at the first back-off stage.

So the successful transimission probability could be described as

$$
P_{s_{-} n_{k}}=\left\{\begin{array}{l}
1, \text { if }\left(n_{k}=1\right) \\
P_{s_{-} n_{k_{-}} \text {backoff }}, \text { if }\left(\mathrm{n}_{k}>1\right)
\end{array},\right.
$$

where $n_{k}=1$ denotes the situation as the Case $1 ;\left(\mathrm{n}_{k}>1\right)$ is related to Case $2 ; P_{s_{-} n_{k_{-}} b a c k o f f}$ is the probability to access channel in first back-off stage as Case 2.

For the Case 2, in view of $(i-1)$ other contending devices, only one device will success in accessing the channel at the first back-off. The probability of a minimum contention window as the first back-off stage is:

$$
\begin{aligned}
& P_{s_{-} n_{k_{-}} \text {backof } f}= \\
& \sum_{k=0}^{W_{\min }-1}\left\{\prod_{0}^{k}\left[1-\frac{1}{W_{\min }}\left(1-\frac{k}{W_{\min }}\right)^{n_{k}-1}\right]\right\} \frac{\left(1-\frac{k+1}{W_{\min }}\right)^{n_{k}-1}}{W_{\min }},
\end{aligned}
$$

where $W_{\min }$ is the minimal size of contention window.

The total number of packets could be sent in one RAW is

$$
\sum_{0}^{M} s_{k}=\sum_{0}^{M} s_{k_{-} 1}+\sum_{0}^{M} s_{k_{-} 2},
$$

where $\sum_{0}^{M} s_{k_{-} 1}$ is the number of packets sent as Case $1 ; \sum_{0}^{M} s_{k_{-} 2}$ is the number of packets sent as Case 2 .

The transmission energy consumption of one RAW is

$$
\begin{aligned}
E_{t}=E_{t 1} \sum_{0}^{M} s_{k_{-} 1}+E_{t 2} \sum_{0}^{M} s_{k_{-} 2} & \\
& +E_{t 3}\left(N-\sum_{0}^{M} s_{k}\right)+E_{c o n} M N,
\end{aligned}
$$

where $E_{t 1}$ is the energy consumption when transmitting a packet successfully as Case $1 ; E_{t 2}$ is the energy consumption when transmitting a packet successfully as Case $2 ; E_{t 3}$ is the energy waste when there is collision when first attempt in selected slot and re-attempt in next slot, so it needs to retransmit in subsequent allocated RAW for this group; $E_{\text {con }}$ is the contention power in one RAW, which is the energy consumed when staying in wake-up mode.

The size of RAW also has the effect on the energy consumption of transmitting overhead information, because when the window size is too small, the overhead information of each device would be high due to the scheduling information that needs to be transmitted multiple times in a short time. And if the number of devices involved in one RAW is small, it also needs massive scheduling information to realize the whole network communication. So the energy consumption of overhead information is related to $N$ and $M$ :

$$
E_{\text {head }}=\frac{\alpha}{M} \times \frac{\beta}{N},
$$

where $\alpha$ is the parameter to indicate the traffic and $\beta$ is the parameter related to overall number of devices in the scenario.

Energy efficiency of one RAW could be evaluated by the data rate it provides and overall energy consumption including transmission energy and overhead energy. Data rate could be formulated as

$$
R=\frac{\sum_{0}^{M} s_{k} \times \gamma}{\tau M},
$$

where $\gamma$ is the packet size and $\tau$ is the time duration of one time slot. $\sum_{0}^{N} S_{j} \times \gamma$ is the total length of packets could be transmitted for $N$ devices in $M$ time slots. $\tau M$ is the total time of one RAW.

The overall energy consumption consists of transition power and overhead power when $N$ devices attempt to communicate with AP during one RAW, which could be denoted by

$$
E_{\text {overall }}=E_{t}+N E_{\text {head }}
$$

Thus energy efficiency is

$$
E E=\frac{R}{E_{\text {overall }}}=\frac{\gamma \sum_{0}^{M} s_{k}}{\tau M\left(E_{t}+N E_{\text {head }}\right)} .
$$

In order to estimate the total number of packets transmitted successfully in one RAW $\left(\sum_{0}^{M} s_{k}\right)$, the average numbers of packets that could be transmitted successfully in each slot are formulated as Equation 11.

Thus energy efficiency is a function related to the number of devices involved $(N)$ and time slot in one RAW $(M)$. We could maximize energy efficiency by finding optimal $M$ based on diverse group size $(N)$. 


$$
\begin{aligned}
& s_{1}=\sum_{\substack{n_{1}=1 \\
N-n_{1}}}^{N} P_{d \_n} P_{s_{-} n_{1}} \\
& s_{2}=\sum_{n_{2}=1}^{N-n_{1}} \sum_{n_{1}=1}^{N}[\underbrace{P_{d_{-} n_{1}}\left(1-P_{s_{-} n_{1}}\right) P_{d_{-} n_{2}}}_{\varepsilon_{21}} P_{s_{-}\left(n_{2}+n_{1}\right)}+\underbrace{P_{d_{-} n_{1}} P_{s_{-} n_{1}} P_{d_{-} n_{2}}}_{\varepsilon_{22}} P_{s_{-}\left(n_{2}+n_{1}-1\right)}] \\
& s_{3}=\sum_{n_{3}=1}^{N-n_{1}-n_{2}} \sum_{n_{2}=1}^{N-n_{1}} \sum_{n_{1}=1}^{N}[\underbrace{\left(\varepsilon_{21}\left(1-P_{s_{-}\left(n_{3}+n_{2}\right)}\right)+\varepsilon_{22}\left(1-P_{s_{-}\left(n_{3}+n_{2}-1\right)}\right)\right) P_{d_{-} n_{3}}}_{\varepsilon_{31}} P_{s_{-}\left(n_{3}+n_{2}\right)} \\
& +\underbrace{\left(\varepsilon_{21} P_{s_{-}\left(n_{3}+n_{2}\right)}+\varepsilon_{22} P_{s_{-}\left(n_{3}+n_{2}-1\right)}\right) P_{d_{-} n_{3}}}_{\varepsilon_{32}} P_{s_{-}\left(n_{3}+n_{2}-1\right)}] \\
& \text {... } \\
& s_{j}=\sum_{n_{j}=1}^{N-\sum_{k=1}^{j-1} n_{k}} \sum_{n_{(j-1)}=1}^{N-\sum_{k=1}^{j-2} n_{k}} \ldots \sum_{n_{1}=1}^{N}[\underbrace{\left(\varepsilon_{(j-1) 1}\left(1-P_{s_{-}\left(n_{j}+n_{(j-1)}\right)}\right)+\varepsilon_{(j-1) 2}\left(1-P_{s_{-}\left(n_{j}+n_{(j-1)}-1\right)}\right)\right) P_{d_{-} n_{j}}}_{\varepsilon_{j 1}} P_{s_{-}\left(n_{j}+n_{(j-1)}\right)} \\
& +\underbrace{\left(\varepsilon_{(j-1) 1} P_{s_{-}\left(n_{j}+n_{(j-1)}\right)}+\varepsilon_{(j-1) 2} P_{s_{-}\left(n_{j}+n_{(j-1)}-1\right)}\right) P_{d_{-} n_{j}}}_{\varepsilon_{j 2}} P_{s_{-}\left(n_{j}+n_{(j-1)}-1\right)}]
\end{aligned}
$$

\section{ENERGY EFFICIENT RAW}

In this section, the energy efficient RAW is devied based on novel retransmission scheme.

The energy efficiency from Section III can be simplified as $E E=$

$$
\gamma(\tau M)^{-1} \sum_{0}^{M} s_{k}
$$

$\overline{E_{t 1} \sum_{0}^{M} s_{k_{-} 1}+E_{t 2} \sum_{0}^{M} s_{k_{-} 2}+E_{t 2}\left(N-\sum_{0}^{M} s_{k}\right)+E_{c o n} N+\frac{\alpha \beta}{M}}$

Let $\xi=\sum_{0}^{M} s_{k}$ and $E_{t_{-} \text {avg }}$ be the average energy consumption to send a packet successfully in a slot, the energy efficiency could be transfer to

$$
E E=\frac{\gamma \xi}{\tau\left[M E_{t \_a v g} \xi+M E_{t 3}(N-\xi)+E_{c o n} M N+\alpha \beta\right]} .
$$

$\xi$ is a function related to $P_{d_{-} n_{k}}$ and $P_{s_{-} n_{k}}$ which can be denoted as $\xi=\sum P_{d_{-} n_{k}} \times P_{s_{-} n_{k}}$, a monotone increasing function along the increment of the number of time slots, because the amount of packets that could be sent increases with increament of the RAW size. For denominator, $M E_{t_{-} a v g} \xi+M E_{t 3}(N-\xi)$ is a decreasing function, since $\xi$ increase with enlarging RAW duration and $E_{t 3} \geq E_{t \_a v g}$. And $E_{c o n} M N$ is a increasing function for $M$, thus denominator is a convex function and the $E E$ is concave with a maximum value.

We find optimal RAW duration for the different group scales based on the novel retransmission scheme to maximize uplink energy efficiency by applying Gradient Descent approach, a fast algorithm to find the optimum of large search space as shown in Algorithm 1.

This algorithm is a standard Gradient Descent approach to find the optimal solution. It starts with an arbitrary value based on a random value, and then compare the existing optimum with a new function result with input that adding a gradient value. When upcoming one performs better, accept new one, otherwise, reserve previous. Step by step to find the optimal value according to gradient descent route.

\section{Simulation RESUlt AND ANALYSis}

In this section, the optimization algorithm for RAW is evaluated in Matlab. We consider a one-hop topology as described in the system model and apply the energy-aware RAW control to the AP and the novel retransmission scheme to collided devices. Since the objective of the RAW control is to optimize energy efficiency and novel retransmission scheme could improve the number of packets sent in a certain time interval, we simulate the energy efficiency and delivery ratio for different group sizes.

We apply wake-up/sleep mode for all devices. At each RAW, there are $N$ devices being allocated. When coming to their RAW, devices keep wake-up mode and randomly select their own time slot to do transmission to the AP, otherwise they would fall into sleep mode. During one RAW, we assume every device has exactly one uplink packet to transmit during one beacon. The main simulation parameters are given in Table I.

TABLE I

SimUlation PARAMETERS

\begin{tabular}{|c|c|c|c|}
\hline Parameter & Value & Parameter & Value \\
\hline frequency & $0.9 \mathrm{GHz}$ & data rate & $100 \mathrm{kbps}$ \\
\hline transmit power for case 1 & $1.346 \mathrm{mw}$ & transmit power for case2 & $2.5 \mathrm{mw}$ \\
\hline collision power & $3.0 \mathrm{mw}$ & idle listen power & $0.001 \mathrm{mw}$ \\
\hline slot duration & $31.1 \mathrm{~ms}$ & packet length & $1024 \mathrm{bits}$ \\
\hline min contention window & 8 & max contention window & 1024 \\
\hline$\alpha$ & 200 & $\beta$ & 200 \\
\hline
\end{tabular}

The energy efficiency for retransmission scheme varying over diverse number of devices based on three different 


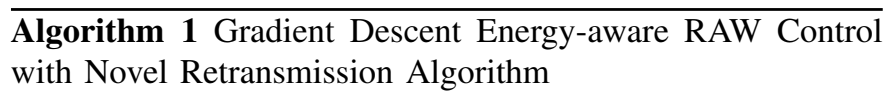

1: Step 1: AP recognizes the size for each group (the number of devices in one group as $N$ ).

2: Step 2: estimate optimal RAW size for a certain group as $M$.

3: loop

4: $\quad$ Initialize $M_{\text {old }}$ and $M_{\text {new }}$ as two random numbers.

5: $\quad E E \_$derivative $(M)=\operatorname{Diff}\{-E E(M, N), M\}$

6: $\quad$ while $a b s\left\{E E\left(M_{\text {old }}\right)-E E\left(M_{\text {new }}\right)\right\} \geq$ precision do

7: $\quad \partial=0.01$

8: $\quad M_{\text {old }}=M_{\text {new }}$

9: $\quad M_{\text {new }}=M_{\text {old }}-\partial \times E E_{\text {derivative }}(M)$

10: end while

11: return $M$

12: end loop

13: Step 3: AP sets the window size as $M$ equal time slots for this group. The devices fall into sleep mode to save energy, and wake up until coming to the allocated window.

14: Step 4: $N$ devices in the group try to estabilish connection with AP by randomly selecting one of $M$ time slots in the window and attempt to access channel.

15: $N$ devices in the group choose one of $M$ slots in the window randomly.

16: Devices attempt to access the channel to build connection with AP as Case 1 and Case 2.

17: When the device could not access in the selected slot, reattempt to access in the following slot. The operation is same as in the previous one.

18: If the device still fail to access the channel in next slot, the device needs to wait for the next allocated RAW for the group to re-access.

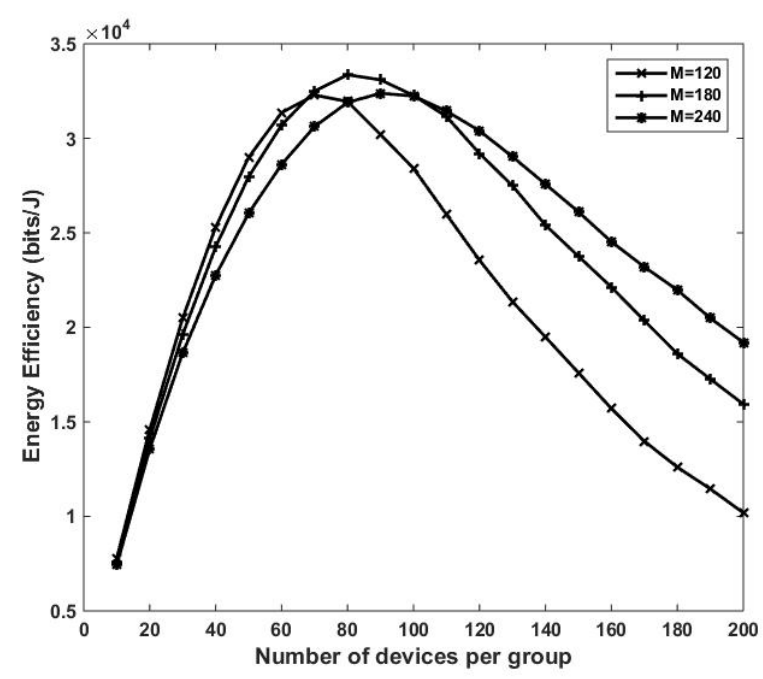

Fig. 4. Energy efficiency comparison based on the same window duration with various amount of devices. durations of one RAW (M=120,180, 240) is shown in Fig. 4. It can be observed that the curves are concave with the one optimal value. The maximum energy efficiency of three window sizes are related to different group sizes. When the number of time slots in RAW is fixed, if the group size is small, energy efficiency for three RAWs goes up with the increment of the number of devices since the overhead energy consumption would decrease for less secheduling information need to send as for whole network communication. And three curves reach the peak points, which are forwarding along the RAW sizes as $\mathrm{N}=70$ for $\mathrm{M}=120, \mathrm{~N}=80$ for $\mathrm{M}=180$ and $\mathrm{N}=100$ for $M=240$, because the optimal capacity of the large RAW is higher than the small one. After the peaks, the trends of three curves declines, and rate of abatement is higher when duration is shorter, since high collision probability lowers the data rate and enlarges energy consumption. It also is the reason to lead to worse performance with short RAW duration for a big group size with high slop in the Fig. 4.

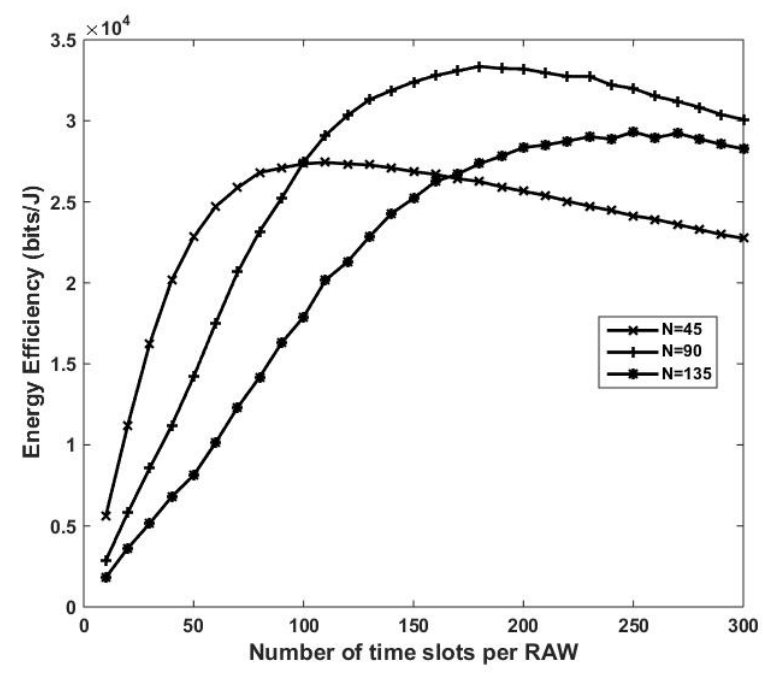

Fig. 5. Energy efficiency comparison based on the same group size with various amount of time slots.

Fig. 5 shows the energy efficiency based on retransmission scheme with three amounts of devices $(\mathrm{N}=45,90,135)$ as it varies along diverse number of time slots. All three curves are concave with a maximum value along the diverse RAW duration. The optimal RAW size for various group sizes is not same. And the best matched window increases with the number of devices per group. When RAW duration is fixed for different groups, if the number of time slots is small, the energy efficiency of three scenarios is low and the trends of the three curves all go up, since collision probabilities are high and decreases with enlarging window size. And that is also the reason for the increasing rates of them are negatively correlated with group scales. At this stage, collision is the main factor for the data rate and energy consumption. The optimal window sizes as the peak points of uplink energy efficiency for three groups increase with the number of devices, for higher capacity for larger window. However, if the RAW 
duration is too large, after the peak value, the energy efficiency would drop due to long time in wake-up mode, which means contention power of devices would be high. The amount of energy consumption on overhead information is opposite with the group size, so the energy efficiency decreasing speed of small group size is higher than that of lager groups.

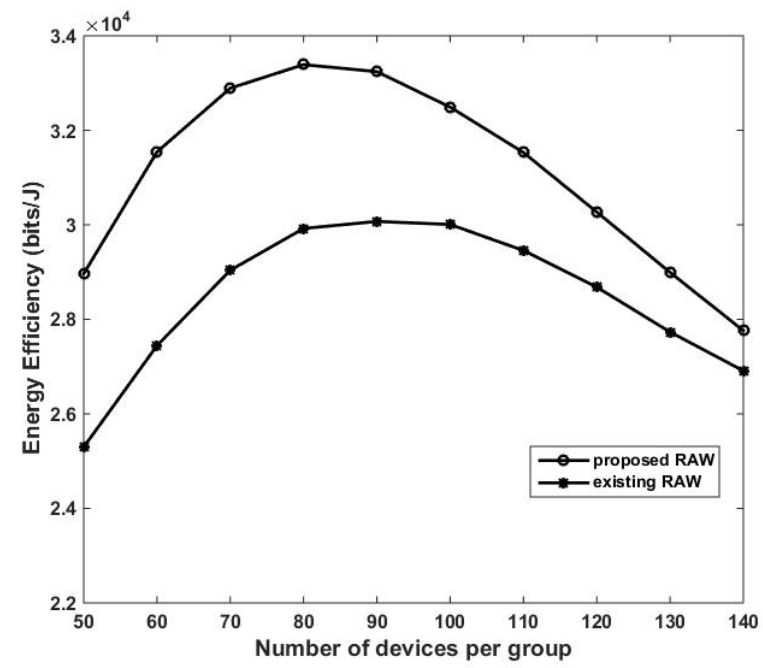

Fig. 6. Energy efficiency comparison between existing RAW and proposed RAW.

Fig. 6 shows the energy efficiency comparison between the existing RAW and the proposed one. The proposed RAW is to set the best matched window size based on group size to achieve optimal energy efficiency based on the novel retransmission scheme that is in this scenario the the collided devices retransmit in next slot, while the legacy scheme is to retransmit in another allocated RAW when there is a collision. According to simulation results, the uplink energy efficiency of proposed RAW improve $12.3 \%$ in general when comparing with the existing RAW.

With random selection in uplink communication, there are many empty slots without device to choose. The proposed RAW with retransmission scheme is to retransmit in next slot for collided devices in crowded slot (selected by more than one device), which could reuse the empty slots, while the exsiting RAW is to retransmit in another allocated RAW, so there are more packets could be transmitted in proposed RAW, leading to high energy efficiency. When the group size is small, the energy efficiency increase along the raising the number of devices, since the scheduling information do not need to send mutiple times which lowers the overhead energy consumption. And large group size brings in more empty time slots in this stage, so the improvement is more distinct with increasing of the number of devices per group. However, the trend declines after peak due to more collision with the high number of devices. And higher active power consumotion for lager group is the other reason for decline.

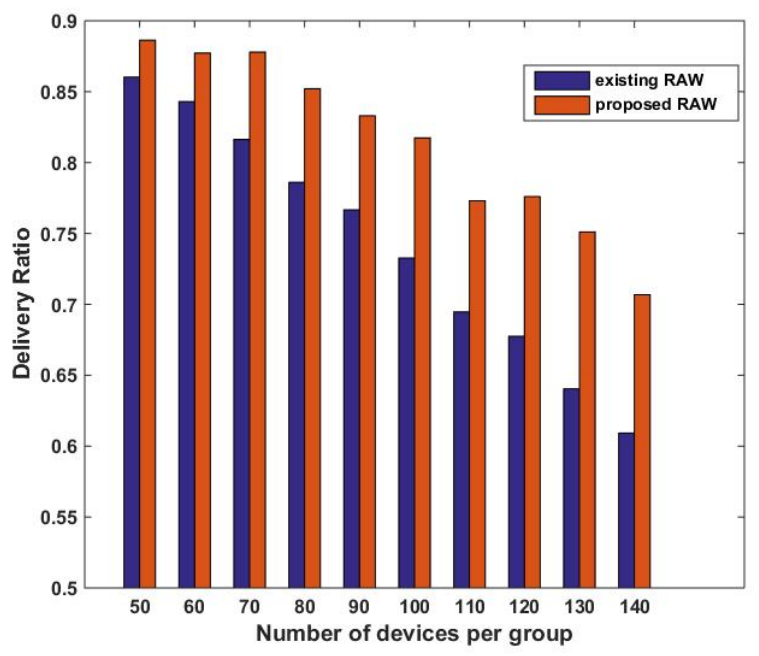

Fig. 7. Delivery Ratio comparison between existing RAW and proposed RAW.

Fig. 7 shows the packet delivery ratio comparison between the existing RAW and the proposed one. It can be observed that the proposed one could improve in general $7.5 \%$ when comparing with the existing RAW. Reuseing empty slots in retransmission scheme makes more packets being sent in one window. In other words, the ratio of the transmitted packets number and the number of time slots is higher for proposed RAW than the existing one. With the rising number of devices per group, the trends of two RAW settings go down due to higher collision probability which leads to consume more energy and less packets that are successfully transmitted. The number of empty time slots in proposed RAW would decrease through retransmission control, so the rate of decrement in proposed one is lower than the existing one and the improvement is larger for higher density of devices per group.

Simulation results demonstrate the proposed energy-aware RAW control with novel retransmission scheme could bring about superior energy efficiency and delivery ratio for IEEE 802.11ah uplink communications.

\section{CONCLUSiOnS}

In this paper, we focus on energy efficiency for IoT applications. The novel retransmission scheme is presented to reuse the empty slots. An energy-aware window control algorithm based on retransmission scheme is proposed for IEEE 802.11ah based networks to optimize uplink energy efficiency through adapting RAW duration for different group sizes for diverse number of devices access simultaneously. The algorithm is built based on Markov Chain and probability theory to monitor the access process and dependency of each time slots. And the transmission probabilities is evaluated of various states a device may fall into when sending a packet during one RAW. On account of that, overall energy consumption and data rate are estimated to contribute to energy efficiency. To maximize the energy efficiency, we find the optimal solution by applying Gradient Descent approach. 
Simulation results demonstrate that the window control based on retransmission scheme could lead to improvement of energy efficiency and delivery ratio for one RAW.

\section{REFERENCES}

[1] A. Gavras, A. Karila, S. Fdida, M. May, M. Potts, "Future internet research and experimentation," ACM SIGCOMM Computer Communication Review, vol. 37, 2007.

[2] ABI Research, (2014, Aug. 20) The Internet of Things Will Drive Wireless Connected Devices to 40.9 Billion in 2020 [Online]. Available: https://www.abiresearch.com/press/the-internet-of-things-willdrivewireless-connect.

[3] A. Zanella, N. Bui, A. Castellani, L. Vangelista and M. Zorzi, Internet of Things for Smart Cities, Internet of Things Journal, IEEE, vol.1, no.1, pp. 22-32, Feb. 2014.

[4] E. Khorov, A. Lyakhov, A. Khorov, A. Guschin, A survey on IEEE 802.11ah: An enabling networking technology for smart cities, Computer Communications (2014)

[5] S. Aust, R. Prasad, I.G.M.M. Niemegeers, "IEEE 802.11ah: Advantages in standards and further challenges for sub $1 \mathrm{GHz}$ Wi-Fi," 2012 IEEE International Conference on Communications (ICC), 2012, pp. 6885 6889.

[6] IEEE Standard for Information Technology Telecommunications and information exchange between systems Local and metropolitan area networks Specific requirements Part 11: Wireless LAN Medium Access Control (MAC) and Physical Layer(PHY) specifications Amendment 10 : Mesh Networking, 2011.

[7] Wi-Fi Alliance. (2016). Wi-Fi Alliance introduces low power, long range Wi-Fi HaLow [Online]. Available: https://www.wi-fi.org/newsevents/newsroom/wi-fi-alliance-introduces-low-power-long-range-wi-fihalow.

[8] A. Hazmi, J. Rinne, M. Valkama, Feasibility study of IEEE 802.11ah radio technology for IoT and M2M use cases, 2012 IEEE Globecom Workshops (GC Wkshps), IEEE, 2012, pp. 1687-1692.

[9] Y. Seok. (2013). Backoff Procedure in RAW [Online]. Available: http://mentor.ieee.org/802.11/dcn/13/11-13-0080-00-00ah-backoffprocedure-in-raw.ppt.

[10] C.W. Park, D. Hwang, T. Lee, Enhancement of IEEE 802.11ah MAC for M2M Communications. Communications Letters, July, 2014, pp. 11511154.

[11] F. Vazquez-Gallego, M. Rietti, J. Bas, J. Alonso-Zarate, L. Alonso, Performance Evaluation of Frame Slotted-ALOHA with Succesive Interference Cancellation in Machine-to-Machine Networks. European Wireless 2014, pp. 403-408

[12] R.P. Liu, G.J. Sutton, I.B. Collings, Power save with offset listen interval for IEEE 802.11ah smart grid communications, 2013 IEEE International Conference on Communications (ICC), IEEE, 2013, pp. 4488-4492.

[13] R.P. Liu, G.J. Sutton, I.B. Collings, WLAN power save with offset listen interval for machine to machine communications, Wireless Communications vol.13 No.5.

[14] Y. Zhou, H. Wang, S. Zheng, Z.Z. Lei, Advances in IEEE 802.11ah standardization for machine-type communications in sub-1GHz WLAN 2013 IEEE International Conference on Communications Workshops (ICC), IEEE, 2013, pp. 1269-1273.

[15] M. Qutab-ud-din, A. Hazmi, B. Badihi, A. Larmo, J. Torsner and M. Valkama, Performance analysis of IoT-enabling IEEE 802.11 ah technology and its RAW mechanism with non-cross slot boundary holding schemes. 2015 IEEE 16th International Symposium on a World of Wireless, Mobile and Multimedia Networks (WoWMoM), IEEE, 2015, pp. $1-6$.

[16] Y. Wang, Y. Li, K. K. Chai, Y. Chen, J. Schormans, Energy-aware Adaptive Restricted Access Window for IEEE 802.11ah Based Networks, 2015 IEEE 26th annual International Symposium on Personal, Indoor and Mobile Radio Communications (PIMRC), IEEE, 2015, pp. 1253-1257.

[17] J. Kim. (2013). RAW Assignment follow up [Online]. Available: http://mentor.ieee.org/802.11/dcn/13/11-13-0510-01-00ah-rawassignment-follow-up.ppt.

[18] Specification Framework for TGah, IEEE Std. 802.11-11 1137r15, May 2013.

[19] E Khorov, A Krotov, A Lyakhov, Modelling Machine Type Communication in IEEE 802.11ah Networks, 2015 IEEE International Conference on Communication Workshop (ICCW), IEEE, May, pp. 1150-1154.
[20] O. Raeesi, J. Pirskanen, A. Hazmi, M. Valkama, Performance evaluation of IEEE 802.11 ah and its reduced access window mechanism, 2014 IEEE International Conference on Communications Workshops (ICC), IEEE, 2014, pp 460-466.

[21] O. Raeesi, J. Pirskanen, A. Hazmi, J. Talvitie and M. Valkama, Performance Enhancement and Evaluation of IEEE 802.11ah Multi-Access Point Network Using Restricted Access Window Mechanism, IEEE International Conference on Distributed Computing in Sensor Systems (DCOSS), 2014, vol., no., pp.287,293, 26-28 May 2014. 